\author{
Gordon D. Rubenfeld \\ Andrew Rhodes
}

\section{How many intensive care beds are enough?}

Received: 5 January 2014

Accepted: 10 January 2014

Published online: 7 February 2014

(C) Springer-Verlag Berlin Heidelberg and ESICM 2014

\section{G. D. Rubenfeld (}

Interdepartmental Division of Critical Care Medicine, University of Toronto Program in Trauma, Emergency and Critical Care, Sunnybrook Health Sciences Center, 2075 Bayview Avenue, Room D108c, Toronto, ON M4N 3M5, Canada e-mail: Gordon.rubenfeld@sunnybrook.ca Tel.: +1-416-4806100

A. Rhodes

St George's Healthcare NHS Trust, St George's University of London, London, UK

One of the most consistent observations in health care is variation. Ever since J. Allison Glover noted a more than fourfold geographic variation in tonsillectomies in England and Wales in 1936 and observed wide swings in procedure rates over time without apparent detrimental effect, researchers have been reporting on the phenomenon. Marked variations in disease incidence, outcome, rates of medical procedures, aggressive care at the end of life, clinician supply and clinical practice are consistently observed and are not fully explained by observable factors such as population demographics [1]. Variation, even within a single country, is so marked that Dartmouth University generates an atlas of health care variation in the US to document the phenomenon [2]. The "differential diagnosis" of this unexplained variation is broad and generally thought to be multifactorial. Likely candidates include uncertainty regarding the optimal management, patient demand, physician enthusiasm for a procedure, available resources for medical care, funding model for health care, malpractice liability and profit motivation [3].

Given this introduction, we would expect there to be variation in critical care services (ICU), and there is $[4,5]$. Not only has variation been documented in the use of procedures where variation might be driven by lack of evidence, for example, in the use of pulmonary artery catheters, tracheostomy and intra-cranial pressure monitors, but there is even marked variation in the decision to admit a patient with a given diagnosis and severity of illness to the ICU [6]. There is also evidence that profit motives and hospital influence drive demand for ICU beds [7]. Importantly, the decision to admit to the ICU is affected by the availability of beds, with a lack of ICU beds leading physicians to appropriately use the available beds for more severely ill patients [8].

The publication in this issue of Intensive Care Medicine by Austin et al. [9] adds to the growing literature on variation in critical care resource supply and demand by expanding the database to middle income and developing countries and by exploring new factors to explain variation. Rather than using the entire population, the authors use deaths due to acute illness as a denominator to reflect burden of disease. This is a novel solution to the fact that we have vanishingly little data on the incidence of critical illness syndromes and almost all of it comes from ICUbased studies that are themselves limited by the number of ICU beds in the studies [10]. In addition, the authors study the relationship between the supply of ICU and hospital beds to gross domestic product (GDP). Here they find that hospital beds, but not ICU beds, seem to be correlated to GDP. We share the authors' caution in interpreting these results. The apparent curvilinear relationship between hospital beds and GDP is driven by a single data point, Boston, which has fewer hospital beds than would be expected from the per capita GDP. This is 
almost certainly due to the US moving much of inpatient care to other settings, in particular, nursing homes and post-acute skilled care facilities leading to a false impression of lower acute care bed needs [11]. Although we might expect an association between ICU beds and GDP, the lack of this association in this analysis should also be viewed cautiously. This is essentially a study with seven observations; therefore, statistical analyses of correlation are problematic. The noise introduced in the analysis by the definition of an ICU bed almost certainly contributes to the ability to exclude a lack of association. More importantly still, there is an important difference between bed supply and access to critical care. This study was only designed to evaluate the former, which is admittedly a crude proxy for access to a skilled multidisciplinary team, surgical resources, laboratory, medication and imaging.

What are we to do with all of these studies on variation in ICU bed supply? The general feeling when confronted with similar data is to assume someone has it right, others have it wrong, and that there are opportunities for efficiencies. This is also likely to be the case with critical care; however, it is much more challenging to address the right number of ICU beds than the right number of tonsillectomies. First, it is impossible to imagine doing a randomized controlled trial for many of the important questions about ICU utilization. Second, the demand for ICU care is driven by every other decision in a health care system; therefore, a focus on the supply of ICU beds will miss the point. How much high-risk surgery is offered? Will intensive chemotherapy be offered at the extremes of indications? What are the cultural norms for end of life care? These factors will drive the demand for intensive care as much as the physiologic indication for life support. Third, the structure of the hospital will lead to the need for more or fewer ICU beds. Will the recovery room fast track extubation for postoperative patients? What is the nurse staffing and training in general beds, and is there a follow-up or rapid response team that can see high-risk ICU discharges so that intensivists are confident with the discharge process? Therefore, the demand for ICU services is likely to vary from hospital to hospital.

These challenges present a tremendous opportunity for health services researchers to identify optimal delivery models but the analyses to account for patient, provider, hospital and system variables are extremely complex. Prospective planning for systems of care that include the ICU is an immature science, and it is unclear whether the lessons from one country or hospital can be transferred to another [12]. It is clear that aging populations plus improving outcomes from critical care and advancing techniques for life support will yield demands for critical care services that some countries will try to meet while others choose to invest elsewhere.

\section{References}

1. Glover JA (1938) The incidence of tonsillectomy in school children: (section of Epidemiology and state medicine). Proc R Soc Med 31(10):1219-1236

2. The Dartmouth Atlas of Health Care (2014). http://www.dartmouthatlas.org/. Accessed 4 Feb 2014

3. Birkmeyer JD, Reames BN, McCulloch P, Carr AJ, Campbell WB, Wennberg JE (2013) Understanding of regional variation in the use of surgery. Lancet 382(9898):1121-1129

4. Wunsch H, Angus DC, Harrison DA et al (2008) Variation in critical care services across North America and Western Europe. Crit Care Med 36(10):2787-2793 (and 2781-2789)
5. Rhodes A, Ferdinande P, Flaatten H, Guidet B, Metnitz PG, Moreno RP (2012) The variability of critical care bed numbers in Europe. Intensive Care Med 38(10):1647-1653

6. Chen LM, Kennedy EH, Sales A, Hofer TP (2013) Use of health IT for highervalue critical care. N Engl J Med 368(7):594-597

7. Marshall MF, Schwenzer KJ, Orsina M, Fletcher JC, Durbin CG Jr (1992) Influence of political power, medical provincialism, and economic incentives on the rationing of surgical intensive care unit beds. Crit Care Med 20(3):387-394

8. Stelfox HT, Hemmelgarn BR, Bagshaw SM et al (2012) Intensive care unit bed availability and outcomes for hospitalized patients with sudden clinical deterioration. Arch Int Med 172(6):467-474
9. Austin S, Srinivas M, Hannah W et al (2013) Access to urban acute care services in high- vs. middle-income countries: an analysis of seven cities. Intensive Care Med. doi: 10.1007/s00134-013-3174-7

10. Adhikari NK, Fowler RA, Bhagwanjee S, Rubenfeld GD (2010) Critical care and the global burden of critical illness in adults. Lancet 376(9749):1339-1346

11. Reinhardt UE (1996) Spending more through 'cost control': our obsessive quest to gut the hospital. Health Aff (Millwood) 15(2):145-154

12. Wild C, Narath M (2005) Evaluating and planning ICUs: methods and approaches to differentiate between need and demand. Health Policy 71(3):289-301 